\title{
Analyse tribo-énergétique du délaminage des couches de revêtements des outils coupants lors d'un usinage à sec des alliages aéronautiques
}

\author{
Mohammed Nouari ${ }^{1, a}$, Hisham Abdel-Aal ${ }^{2}$, Mohamed El Mansori ${ }^{3}$ \\ et Armansyah Ginting ${ }^{4}$ \\ 1 Laboratoire Matériaux Endommagement Fiabilité Ingénierie des Procédés (LAMEFIP), ENSAM CER Bordeaux, \\ Esplanade des Arts et Métiers, 33405 Talence, France \\ 2 Department of General Engineering, 151 Ottensman Hall, University of Wisconsin-Platteville, 1 University Plaza, Platteville, \\ WI 53818-3099, USA \\ ${ }^{3}$ Laboratoire de Mécanique et Procédés de Fabrication (LMPF), ENSAM CER Châlons-en-Champagne, Rue Saint Dominique \\ BP 508, 51006 Châlons-en-Champagne, France \\ 4 Department of Mechanical Engineering, Faculty of Engineering, University of Sumatera Utara, Jalan Almamater, \\ 20155 Medan, North Sumatera, Indonesia
}

Reçu le 21 août 2006, accepté le 9 février 2007

\begin{abstract}
Résumé - Le chargement thermomécanique imposé lors d'une opération de coupe engendre une usure importante des outils coupants, plus particulièrement lorsque l'usinage est réalisé sur des superalliages réfractaires à base de nickel et/ou de titane. Le transfert de la chaleur générée par le frottement intense à l'interface outil-copeau joue un rôle primordial dans la préservation de l'intégrité de l'outil. Ce transfert dépend essentiellement des propriétés thermiques et de leur évolution en fonction de la température et des autres paramètres mécaniques du matériau (déformation, vitesse de déformation, etc.). Dans cette étude, l'effet des propriétés thermiques sur l'usure produite pendant l'usinage des alliages réfractaires est analysé. L'utilisation d'une analyse par éléments-finis couplée à des observations microscopiques des modes d'endommagement ont permis de suivre l'évolution de la conductivité thermique dans la zone de l'outil affectée par l'usure. Les résultats obtenus montrent une réduction importante de la conductivité thermique pendant l'écoulement du copeau. Cette réduction limite localement et de façon très importante la capacité du matériau à dissiper la charge thermique appliquée. Plusieurs zones apparaissent alors aux niveaux des faces de coupe et de dépouille de l'outil où la conductivité atteint des niveaux très bas, (zones mortes thermiquement). Par conséquent, différents mécanismes d'usure apparaissent et affectent la résistance à la microfissuration du substrat de l'outil et au délaminage de son revêtement.
\end{abstract}

Mots clés : Usinage à sec / usure / délaminage du revêtement / température de coupe / propriétés thermiques

Abstract - Triboenergetic analysis of coating layers delamination when dry machining aero-
nautical alloys. The applied thermo-mechanical loading during cutting process leads to an important
tool failure especially when machining is effected on refractory super-alloys such as titanium and/or
nickel based-alloys. Heat removal generated by tool/workpiece friction plays an important role in pre-
serving the structural integrity of the cutting tool. Efficient heat removal in machining depends solely
on the thermal properties of the tool and on their evolution with temperature and mechanical pa-
rameters (strain, strain rate, etc.). In the present work, the effect of these thermal properties on the
produced wear during the machining of refractory alloys is particularly investigated. Finite-Element
Analysis (FEA) and SEM-imagery were used to map the thermal conductivity within the affected tool
zone. The results indicate that depending on the temperature rise, the tool-tip might undergo a se-
vere drop in thermal conduction. This drop may locally restrict the ability of the tool material to
dissipate the applied thermal load. This may nucleate thermally congested clusters within the tool-tip

a Auteur correspondant :

mohammed.nouari@lamef. bordeaux.ensam.fr 
where the material completely loses the ability to transport heat. Thermal congestion renders an energetically active zone where the thermal energy available may be used to activate wear through different mechanisms. It is also found that the immediate layer under the surface of the tool tip is important to enhance the ability of the tool material to dissipate the thermal loads. The results also highlight the importance of matching the temperature dependant properties of the different coating layers in order to enhance delamination resistance.

Key words: Machining / wear / coating delamination / cutting temperature / thermal conductivity

\section{Introduction}

Actuellement, l'usinage à sec ou avec lubrification contrôlée (micro-lubrification) suscite un intérêt accru de la part des différents gouvernements européens. Dans un souci de prévention de la pollution et du respect de l'environnement, une pression croissante est exercée sur les industriels pour diminuer - voire supprimer - la quantité de lubrifiants utilisée. L'usinage à sec se présente alors comme une solution alternative face aux problèmes de pollution que connaît la planète. Dans un premier temps, l'usinage à sec a commencé à se développer pour des raisons de coût. Plusieurs études révèlent que les fluides de coupe représentent environ $16 \%$ des coûts d'usinage d'une pièce. L'absence de la lubrification engendre une importante élévation de la température de coupe qui implique systématiquement une perte de précision sur les pièces finies et une détérioration prématurée des arêtes de coupe. Pour remédier à ce dernier problème, les fabricants d'outils ont souvent recours à des revêtements (mono ou multicouches) de type $\mathrm{Al}_{2} \mathrm{O}_{3}$, TiC, TiN, etc. [1-7]. Il subsiste cependant plusieurs difficultés techniques à contourner afin d'améliorer la résistance aux problèmes de délaminage des revêtements et ainsi optimiser complètement la tenue à l'usure des outils coupants.

Le délaminage est un mode d'endommagement assez fréquent; parfois très localisé au voisinage de l'arête de coupe, il est souvent observé lors de l'usinage à sec des alliages aéronautiques. Les études précédentes $[8,9]$ montrent que l'origine de ce type d'endommagement est assez difficile à appréhender. Ceci est essentiellement dû à l'interaction complexe entre phénomènes mécaniques et physico-chimiques. Lors de la coupe de l'alliage de titane Ti-6Al-4V par exemple, la forte réactivité chimique vis-à-vis d'une grande majorité d'outils carbures fait que le phénomène d'adhésion à l'interface outilcopeau est le principal précurseur du délaminage [9]. La déformation importante du matériau au niveau de l'interface outil-copeau contribue également à l'amplification du délaminage $[8,9]$. La déformation plastique, indicateur d'une pression de contact élevée, modifie de façon significative la géométrie de l'arête de coupe et augmente considérablement les dimensions de la zone de contact. La température élevée dans cette zone mène généralement à la dégradation des propriétés thermiques et mécaniques du triplet tribologique «outil/revêtement/pièce ». Cette dégradation des propriétés thermiques affecte la résistance du matériau de l'outil à la microfissuration et au délaminage de son revêtement. Par ailleurs, une température élevée génère aussi un
Tableau 1. Composition chimique de l'alliage de titane Ti6242S (wt\%).

\begin{tabular}{ccc}
\hline Éléments & Min $(\%)$ & Max $(\%)$ \\
\hline $\mathrm{Al}$ & 5,50 & 6,50 \\
$\mathrm{Zr}$ & 3,60 & 4,40 \\
$\mathrm{Mo}$ & 1,80 & 2,20 \\
$\mathrm{Sn}$ & 1,80 & 2,20 \\
$\mathrm{Fe}$ & - & 0,25 \\
$\mathrm{O}_{2}$ & - & 0,15 \\
$\mathrm{Si}$ & - & 0,10 \\
$\mathrm{C}$ & - & 0,05 \\
$\mathrm{~N}_{2}$ & - & 0,05 \\
$\mathrm{H}_{2}$ & - & 0,015 \\
$\mathrm{Y}$ & - & 0,005 \\
autres & 0,100 & 0,300 \\
\hline
\end{tabular}

Tableau 2. Propriétés mécaniques et thermiques de l'alliage de titane Ti-6242S (wt\%).

\begin{tabular}{ll}
\hline Limite élastique en traction (MPa) & $\geq 895$ \\
Limite élastique en compression (MPa) & 830 \\
Contrainte de fluage (MPa) & 240 \\
Dureté (HRe) & $\sim 36$ \\
Masse volumique $\left(\mathrm{kg} \cdot \mathrm{m}^{-3}\right)$ & 4540 \\
Expansion thermique linéaire $\left(10^{-6} \cdot{ }^{\circ} \mathrm{C}^{-1}\right)$ & 9,9 \\
Conductivité thermique $\left(\mathrm{W} \cdot \mathrm{mK}^{-1}\right)$ & $8-12$ \\
\hline
\end{tabular}

flux de chaleur intense. Ce dernier constitue une source d'énergie supplémentaire et accélère la diffusion chimique des constituants de l'outil, du revêtement et de la pièce usinée. Partant de ce constat, l'identification de la relation «propriétés thermiques/usure » est primordiale dans la caractérisation des différents mécanismes responsables du processus de délaminage.

\section{Essais d'usure et conditions d'usinage à sec}

Différents essais de fraisage à sec ont été effectués précédemment sur l'alliage de titane Ti-6242S de structure $\alpha \beta[8,9]$, voir tableaux 1 et 2 . Deux groupes d'outils carbure ont été choisis : le premier de type mixte et de désignation $\mathrm{WC}-\mathrm{Ti} / \mathrm{Ta} / \mathrm{Nb}-\mathrm{Co}$ ne comporte aucun revêtement. Il est pris comme le matériau de référence. Le second est du même type avec 9 couches de revêtement empilées sur le substrat de la façon suivante : $\mathrm{TiN} / \mathrm{TiC} / \mathrm{TiN} / \mathrm{TiC} / \mathrm{TiN} / \mathrm{TiC} / \mathrm{TiN} / \mathrm{TiCN} / \mathrm{TiN}$. L'épaisseur totale du revêtement est de l'ordre de $10 \mu \mathrm{m}$, sa composition chimique ainsi que ses propriétés thermiques et mécaniques sont présentées respectivement 
Tableau 3. Composition chimique et propriétés mécaniques et thermiques de l'outil carbure (substrat).

\begin{tabular}{|c|c|}
\hline Caractéristiques du substrat de l'outil & $\begin{array}{c}\text { Outils A } \\
\text { et B }\end{array}$ \\
\hline \multirow{4}{*}{ Composition chimique } & $69,8 \% \mathrm{WC}$ \\
\hline & $9,50 \% \mathrm{Co}$ \\
\hline & $20,7 \%$ \\
\hline & $(\mathrm{Ti} / \mathrm{Ta} / \mathrm{Nb})$ \\
\hline Taille du grain $(\mu \mathrm{m})$ & $1-2$ \\
\hline Dureté à $25^{\circ} \mathrm{C}\left(\mathrm{HV}_{10}\right)$ & 1485 \\
\hline Dureté à chaud $800^{\circ} \mathrm{C}\left(\mathrm{kg} \cdot \mathrm{mm}^{-2}\right)$ & 600 \\
\hline Masse volumique $\left(\mathrm{g} \cdot \mathrm{cm}^{-3}\right)$ & 11,4 \\
\hline Conductivité thermique (W.mK ${ }^{-1}$ ) & 45 \\
\hline Expansion thermique $\left(10^{-6} \mathrm{~K}^{-1}\right)$ & 6,1 \\
\hline Module d'élasticité (GPa) & 510 \\
\hline Contrainte de rupture (GPa) & 2,2 \\
\hline
\end{tabular}

dans les tableaux 3 et 4 . L'examen au profilomètre optique révèlent des rugosités de surface $R_{\mathrm{a}}$ de l'ordre de $0,5 \mu \mathrm{m}$ et $R_{\mathrm{t}}$ de l'ordre de $5 \mu \mathrm{m}$.

Dans le procédé de fraisage-boule, la géométrie de l'outil est définie par les paramètres suivants : l'angle de coupe principal $\gamma_{0}=-6^{\circ}$, l'angle de coupe axial $\gamma_{\mathrm{p}}=-6^{\circ}$ et radial $\gamma_{\mathrm{f}}=-2^{\circ}$. Les autres conditions de coupe sont : la vitesse de coupe $V$ prise dans une gamme allant de 100 à $125 \mathrm{~m} \cdot \mathrm{min}^{-1}$, l'avance par dent $f_{z}$ de 0,15 à $0,20 \mathrm{~mm}$.dent ${ }^{-1}$ et la profondeur de coupe axiale $a_{\mathrm{a}}$ de 2 à $2,5 \mathrm{~mm}$. La profondeur de coupe radiale $a_{\mathrm{r}}$ est fixée dans tous les essais à $8,8 \mathrm{~mm}$ [10].

\section{Identification des mécanismes d'usure}

\subsection{Outils non revêtus}

Une analyse par microscopie électronique est systématiquement réalisée sur les outils neufs (avant usinage) et l'ensemble des outils endommagés. Le critère d'usure adopté dans les essais (durée de vie de l'outil) est celui fixant l'usure en dépouille $V_{\mathrm{B}}$ à une valeur limite de l'ordre de 0,3 mm, norme ISO 8688-2 [11]. Aussi, l'outil est retiré dans le cas d'un ébrèchement excessif de la face de coupe, d'un écaillage et/ou d'une rupture de l'arête de coupe [11].

La figure 1 présente une micrographie de l'arête principale de l'outil non revêtu pour lequel l'usure en dépouille $V_{\mathrm{B}}$ a atteint la valeur limite fixée au préalable par le critère. En confrontant le profil de l'outil usé à celui de l'outil neuf, (ligne de tirés blancs sur la Fig. 1a), une rupture fragile, un écaillage ainsi qu'un ébrèchement sont clairement identifiés (voir Fig. 1b). En raison d'un écaillage important de l'arête de coupe, le mécanisme de fissuration n'a pas pu être observé. Le mode d'usure par adhésion est mis en évidence pour ce même outil sur la figure 2. En fait, lors de l'écoulement du copeau à la surface de l'outil, la température, le frottement ainsi que la pression de contact sont particulièrement élevés, des soudures se forment alors entre les deux matériaux antagonistes.

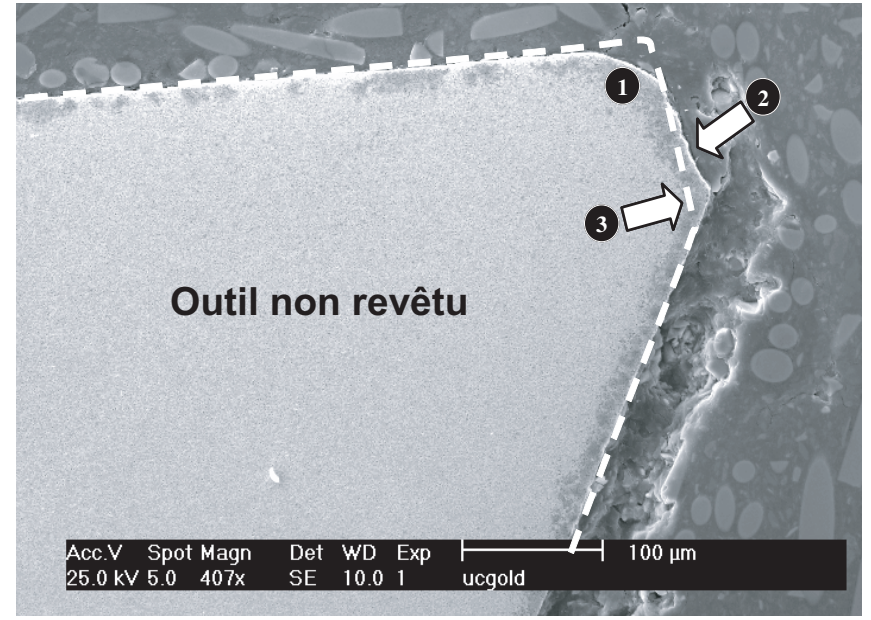

(a)

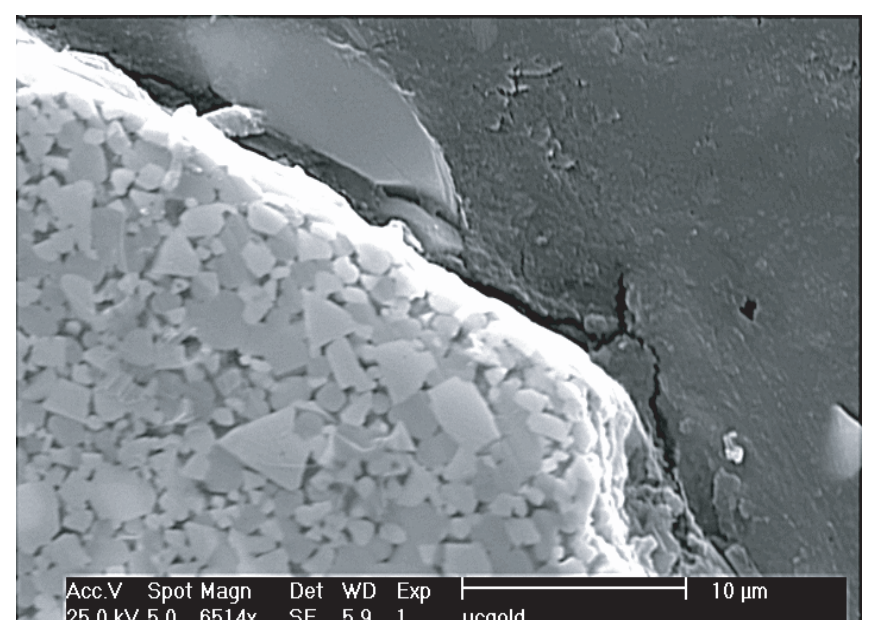

(b)

Fig. 1. Micrographies de l'outil non revêtu. Conditions de coupe : $V_{\mathrm{c}}=100 \mathrm{~m} \cdot \mathrm{min}^{-1}, f_{z}=0,15 \mathrm{~mm} \cdot \mathrm{dent}^{-1}, a_{\mathrm{a}}=2 \mathrm{~mm}$, $a_{\mathrm{r}}=8,8 \mathrm{~mm}$. (a) Rupture fragile, la ligne de tirets blancs désigne le profil initial de l'outil neuf, (b) grossissement de la zone affectée. (1) Écaillage, (2) ébrèchement, (3) déformation plastique.

Sous l'effet du chargement mécanique appliqué au niveau du contact, ces soudures se rompent provoquant ainsi un écaillage de l'arête de coupe. Ceci montre que l'adhésion est le mécanisme d'endommagement primaire. Il s'en suit alors les autres mécanismes constatés tels que la rupture d'arête, l'ébrèchement et la cratérisation. La formation des couches d'adhésion lors de l'usinage des alliages de titane est essentiellement due à la réactivité chimique de ce matériau vis-à-vis de celui de l'outil [12] . En effet, l'absence d'une barrière empêchant la diffusion (couche de revêtement) facilite la circulation des éléments chimiques de part et d'autre de l'interface. Sous l'effet supplémentaire de la température et de la pression, des réactions chimiques se produisent et dégradent l'intégrité de surface de l'arête de coupe. L'analyse chimique de la couche d'adhésion formée confirme que les grains situés à 
Tableau 4. Propriétés mécaniques et thermiques des couches de revêtement.

\begin{tabular}{lc}
\hline Caractéristiques des revêtements & Outil B \\
\hline Méthode de déposition & $\mathrm{CVD}:$ Chemical Vapour Deposition \\
Ordre d'empilements des couches de & 9 couches : \\
revêtement (de l'intérieur vers l'extérieur) & $\mathrm{TiN} / \mathrm{TiC} / \mathrm{TiNfTiC} / \mathrm{TiN} / \mathrm{TiC} / \mathrm{TiN} / \mathrm{TiCN} / \mathrm{TiN}$ \\
Épaisseur totale & $=10 \mu \mathrm{m}$ \\
Type de matériau du revêtement & $\mathrm{TiN}, \mathrm{TiC}, \mathrm{TiCN}$ \\
Dureté à $25^{\circ} \mathrm{C}\left(\mathrm{HV} \mathrm{V}_{10}\right)$ & $2200,2110,2300$ \\
Conductivité thermique à $727^{\circ} \mathrm{C}\left(\mathrm{W} \cdot \mathrm{mK}^{-1}\right)$ & $25,28,31$ \\
Expansion thermique $\left(10^{-6} \mathrm{~K}^{-1}\right)$ & $9,35,8,85,8,65$ \\
Température de fusion $\left({ }^{\circ} \mathrm{C}\right)$ & $2950,3000,3070$ \\
Masse volumique $\left(\mathrm{g} \cdot \mathrm{cm}^{-3}\right)$ & $3,44,3,65,4,18$ \\
\hline
\end{tabular}

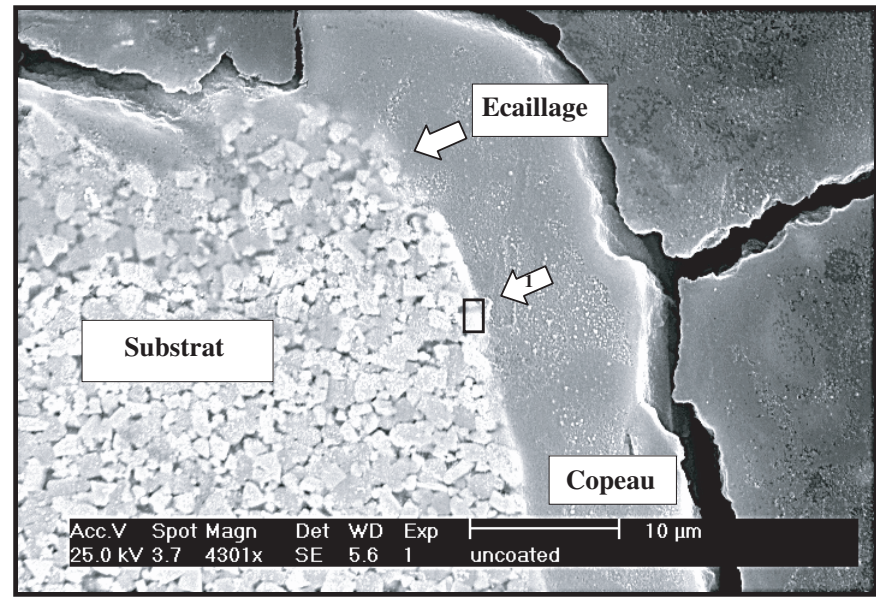

Fig. 2. Usure par adhésion de l'outil non revêtu. Les conditions de coupe sont : $V_{\mathrm{c}}=100 \mathrm{~m} \cdot \mathrm{min}^{-1}, f_{z}=0,15 \mathrm{~mm} \cdot \mathrm{dent}{ }^{-1}$, $a_{\mathrm{a}}=2 \mathrm{~mm}, a_{\mathrm{r}}=8,8 \mathrm{~mm}$. L'analyse chimique de l'interface substrat-copeau est donnée par le tableau 5 .

proximité de l'interface contiennent des constituants des deux matériaux en contact (tungstène $\mathrm{W}$, carbone $\mathrm{C}$, cobalt Co, titane Ti, etc.), cf. figure 2 et tableau 5 .

\subsection{Outils revêtus}

Dans le cas des outils revêtus, l'écaillage, la fissuration et la déformation plastique sont les principaux modes d'endommagement des arêtes de coupe (cf. Fig. 3). La ligne de tirets noirs sur la figure 3 représente le profil initial de l'outil (avant usinage). La déformation plastique importante observée montre la sévérité du chargement mécanique appliqué pendant l'opération d'usinage des alliages de titanes (matériaux à faible usinabilité). Les micrographies de la figure 3 montrent aussi un phénomène de fissuration au sein du revêtement multicouches. Le suivi de l'usure pendant la formation du copeau a permis de déduire que la propagation des fissures se fait simultanément dans le sens horizontal (sens parallèle à l'écoulement du copeau) et vertical (sens perpendiculaire), donnant ainsi lieu à un délaminage au niveau de l'interface substrat/revêtement, voir figure $3 \mathrm{~b}$.
Tableau 5. Analyse chimique de l'interface outil non revêtu (substrat)/copeau.

\begin{tabular}{lcc}
\hline Élements chimiques & Symbole & $\mathrm{Wt}(\%)$ \\
\hline Tungsten & $\mathrm{W}$ & 40,01 \\
Titanium & $\mathrm{Ti}$ & 3,14 \\
Tantalum & $\mathrm{Ta}$ & 7,25 \\
Niobium & $\mathrm{Nb}$ & 15,09 \\
Carbon & $\mathrm{C}$ & 5,59 \\
Cobalt & $\mathrm{Co}$ & 1,15 \\
\hline
\end{tabular}

\section{Analyse du processus de délaminage du revêtement}

\subsection{Initialisation de l'usure des outils de coupe}

Afin d'analyser l'initiation de l'usure, des essais supplémentaires ont été conduits sur les outils revêtus. Pour chaque essai, une seule passe a été autorisée sur la pièce. La longueur usinée est d'environ $250 \mathrm{~mm}$. Les outils usés ont été sectionnés et examinés à l'endroit où le délaminage a été localisé. Comme le montre la figure 4b, les couches de revêtement enlevées au substrat sont clairement identifiées. D'autres investigations ont montré que le délaminage est le mode d'endommagement initial; il se produit généralement après quelques secondes d'usinage seulement. Dans un premier temps, il peut être supposé que le délaminage est d'origine mécanique (usure par abrasion). Cependant, les efforts de coupe enregistrés lors de l'usinage des alliages de titane ne sont pas très élevés et approximativement similaires à ceux mesurés pour les aciers doux [6]. En général, les revêtements de type $\mathrm{TiN}, \mathrm{TiC}$ et $\mathrm{TiCN}$ améliorent le frottement et la durée de vie des outils dans le cas de l'usinage des aciers $[1,5,6]$. Cette tendance n'est pas conservée pour les alliages de titane. Pour ces derniers, le chargement cyclique appliqué (mécanique et thermique) affaiblit la résistance du revêtement aux chocs à chaque engagement de l'outil dans la matière.

\subsection{Phénomènes physiques à l'origine du délaminage des couches de revêtement}

Selon les observations expérimentales présentées précédemment, une description détaillée des mécanismes 


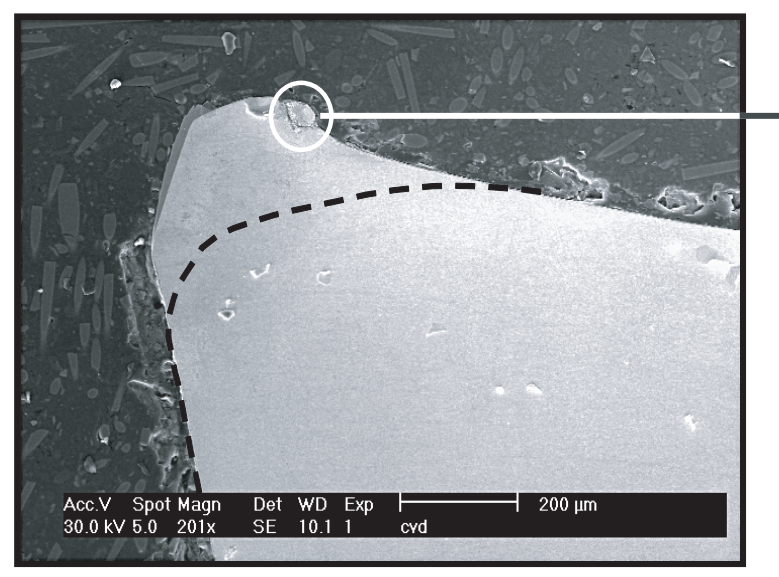

(a)

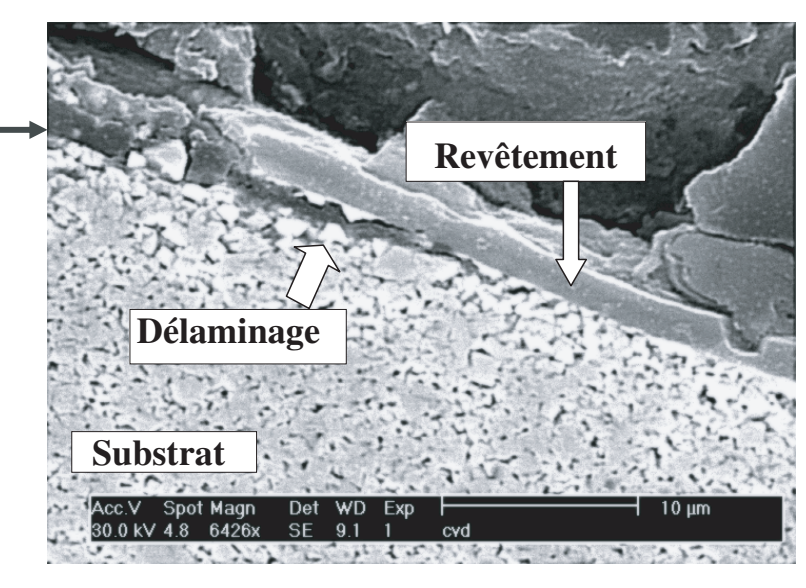

(b)

Fig. 3. Mécanismes d'usure d'un outil carbure revêtu. (a) Déformation plastique de l'arête de coupe. (b) Grossissement de la partie d'outil entourée de tirets noirs sur la figure 3a.

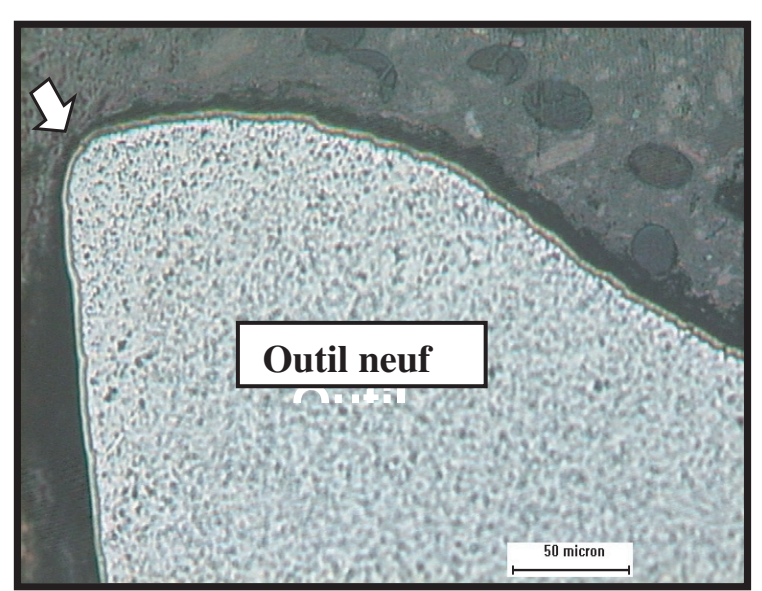

(a)

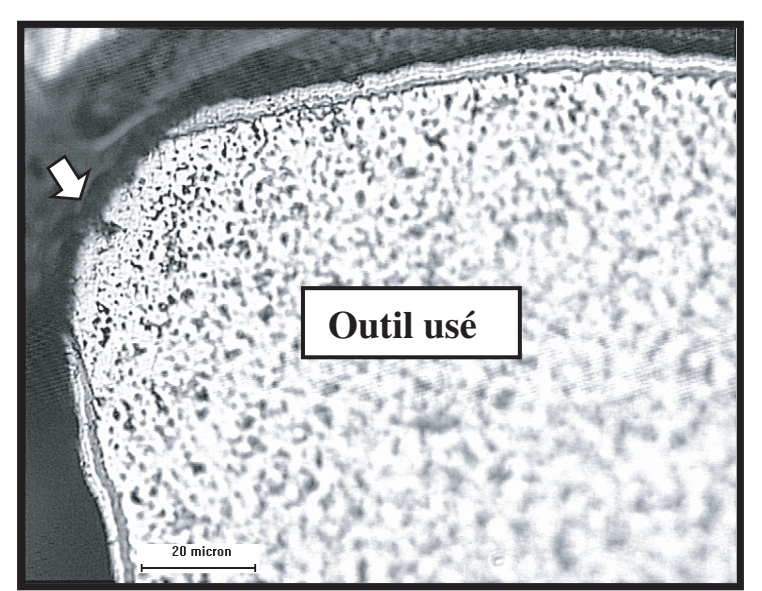

(b)

Fig. 4. Mécanisme de délaminage du revêtement. (a) Outil neuf. (b) Outil usé.

physiques à l'origine du délaminage est faite dans la suite de ce paragraphe. L'analyse des modes d'endommagement a montré que l'interface outil-copeau est essentiellement contrôlée par la pression et la température de contact. Cette dernière peut atteindre des valeurs significatives (de l'ordre de $1000^{\circ} \mathrm{C}$ ) et affecter ainsi le comportement thermique et mécanique du revêtement. Des travaux antérieurs $[9,13]$ ont confirmé que les températures élevées dans le contact outil-copeau sous haute pression favorisent la constitution d'un environnement idéal pour l'usure chimique (dissolution, diffusion, etc.).

La distribution de la température a été calculée numériquement dans les mêmes conditions d'essais expérimentaux. Les résultats obtenus sont montrés sur la figure 5. Le calcul de la température a été réalisé pour les deux types d'outils de l'étude : l'outil A = outil non revêtu et l'outil $\mathrm{B}=$ outil revêtu. Des températures maximales de l'ordre de $1150{ }^{\circ} \mathrm{C}$ pour l'outil B, et de l'ordre de $950{ }^{\circ} \mathrm{C}$ pour l'outil $\mathrm{A}$ sont localisées à quelques dizaines de micromètres de la pointe de l'outil. Ces températures élevées témoignent aussi de la présence d'une grande déformation plastique à proximité de la pointe comme le montre la figure 3a. La combinaison d'une déformation plastique extrême et d'un frottement intense à l'interface outilcopeau engendre une source de chaleur supplémentaire au niveau de la surface de contact. La capacité du matériau à dissiper la chaleur dans cette zone est alors affectée [14].

Comme représenté schématiquement sur la figure 5, la localisation du maximum de température génère deux flux thermiques notés $q_{2 \mathrm{~A}}$ pour l'outil A (non revêtu) et $q_{2 \mathrm{~B}}$ pour l'outil B (revêtu); le premier est orienté du point où la température est maximale (point II pour l'outil $\mathrm{A}$ et I pour l'outil B) vers le substrat. Le second gradient est orienté du même point vers la surface de contact. Ces deux flux thermiques dépendent fortement de la valeur locale de la conductivité thermique aux points I et II. De plus, pour l'outil revêtu B, ces flux dépendent aussi de la conductivité thermique effective de la combinaison des différentes couches de revêtement. 


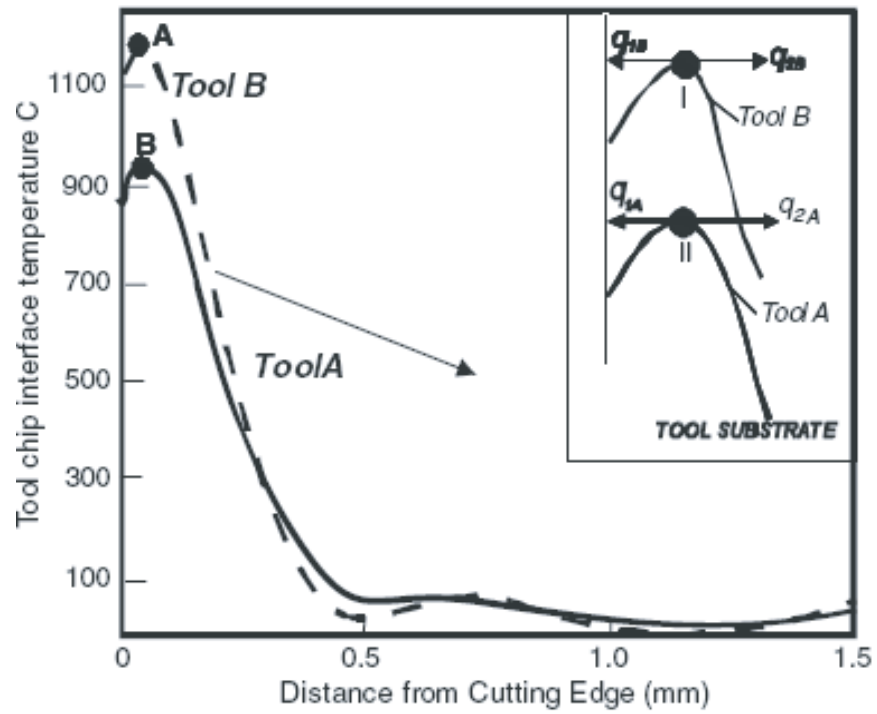

Fig. 5. Évolution de la température à la surface de l'outil non revêtu (outil A) et à celle de l'outil revêtu (outil B).

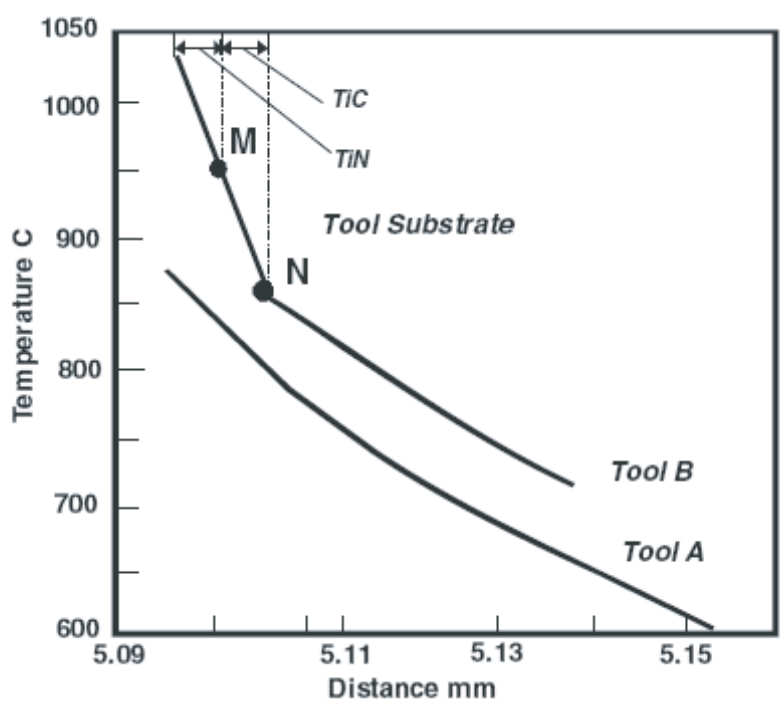

Fig. 6. Évolution de la température aux différentes interfaces : outil A (outil non revêtu)/copeau, outil B (outil revêtu)/copeau. Les points $\mathrm{M}$ et $\mathrm{N}$ pour l'outil $\mathrm{B}$ désignent respectivement les interfaces $\mathrm{TiN} / \mathrm{TiC}$ et $\mathrm{TiC} /$ substrat.

Comme illustré sur la figure 6, la température à l'interface des deux couches de revêtement $\mathrm{TiN} / \mathrm{TiC}$ est de l'ordre de $950{ }^{\circ} \mathrm{C}$ (point $\mathrm{M}$ sur la Fig. 6). À cette température, le coefficient d'expansion thermique $\alpha$ de l'élément $\mathrm{TiC}$ est de $8,47 \mathrm{ppm} .{ }^{\circ} \mathrm{C}$ tandis que celui du TiN n'est que de $6,35 \mathrm{ppm} .{ }^{\circ} \mathrm{C}$, soit un rapport $\alpha(\mathrm{TiC}) / \alpha$ (TiN) de l'ordre de 1,4. Cette différence entre coefficients d'expansion thermique génère lors de séquences de refroidissement et de chauffage, (comme c'est le cas du chargement cyclique en usinage), des contraintes thermiques aux niveaux des interfaces. Ceci explique en partie l'origine du délaminage entre les deux premiers constituants du revêtement $\mathrm{TiC}$ et $\mathrm{TiN}$. Un autre délaminage peut également se produire au niveau du contact : substrat $/ 1^{\mathrm{e}}$ couche du revêtement. En effet, des contraintes au niveau de ce contact sont systématiquement créées à cause de la variation de volume entre les matériaux en contact (substrat et couches de revêtement). Connues sous le nom de « contraintes de croissance », elles sont à l'origine de la fissuration et de la propagation de microfissures dans le matériau. Il est très important de noter ici que les caractéristiques mécaniques et thermiques des couches de revêtement sous forme de couches minces et sous forme d'un massif sont complètement différentes (massif $\approx 10 \mu \mathrm{m}$ ).

La résistance d'un matériau à développer des microfissures est une fonction des propriétés mécaniques et thermiques ainsi que des conditions du transfert thermique. Elle est définie classiquement par la relation suivante :

$$
R=\frac{\sigma_{\mathrm{th}}}{E \alpha B}
$$

où $\sigma_{\text {th }}$ la contrainte thermique dans le matériau, $E$ le module d'Young, $\alpha$ le coefficient d'expansion thermique et $B$ le nombre de Biot. Ce dernier paramètre dont la forme est donnée par l'équation (2), est un nombre sans dimensions qui compare les résistances au transfert de chaleur à l'intérieur et à la surface d'un échantillon. Dans le cas des systèmes « minces », $B$ est très faible car la température peut être considérée comme constante au sein du corps mince. Pour les systèmes dits « épais », $B$ est d'ordre un.

$$
B=\frac{h L_{\mathrm{C}}}{K}
$$

Dans l'équation (2) $h$ est le coefficient global de transfert de chaleur et $L_{\mathrm{C}}$ est une longueur caractéristique habituellement définie comme le volume de l'échantillon divisé par sa surface. $K$ est la conductivité thermique. Dans cette étude, $L_{\mathrm{C}}$ et $h$ sont considérés constants pour les revêtements utilisés. En combinant les équations (1) et (2), le rapport des résistances à la microfissuration de deux matériaux I et II s'exprime de la façon suivante :

$$
\frac{R_{\mathrm{I}}}{R_{\mathrm{II}}}=\frac{\sigma_{\mathrm{th}_{\mathrm{I}}} E_{\mathrm{II}} \alpha_{\mathrm{II}} K_{\mathrm{I}}}{\sigma_{\text {th_II }} E_{\mathrm{I}} \alpha_{\mathrm{I}} K_{\mathrm{II}}}
$$

Les valeurs des paramètres thermiques correspondant aux différents revêtements utilisés dans cette étude sont regroupés dans le tableau 6 . Le rapport de l'équation (3) appliqué aux revêtements $\mathrm{TiN}$ et $\mathrm{TiC}$ montre une variation de 0,97 à 1,2 pour une température de $950{ }^{\circ} \mathrm{C}$ au point $\mathrm{M}$ de la figure 6 (interface $\mathrm{TiN} / \mathrm{TiC}$ ). $\mathrm{Au}$ point $\mathrm{N}$ (interface $\mathrm{TiC} /$ substrat), pour une température de $850{ }^{\circ} \mathrm{C}$, ce rapport n'est que de 0,5. Ceci montre qu'avec cette configuration d'empilement de couches de revêtement, l'élément $\mathrm{TiC}$ présente une plus faible liaison avec le substrat qu'avec l'élément TiN. Il en résulte une plus forte probabilité d'un délaminage au niveau de l'interface substrat/revêtement (point M) qu'au niveau de l'interface TiN/TiC (point N). Une solution peut alors être proposée en modifiant la séquence d'empilement pour obtenir un rapport de résistances à la microfissuration 
Tableau 6. Propriétés des différents matériaux aux niveaux des interfaces TiN/TiC (point M de la Fig. 6) et TiC/Substrat (point $\mathrm{N}$ de la Fig. 6).

\begin{tabular}{cccccc}
\hline Matériau & $V h(\mathrm{GPa})$ & $E(\mathrm{GPa})$ & $k\left(\mathrm{w} / \mathrm{m}^{\circ} \mathrm{C}\right)$ en $\mathrm{TM}$ & $k\left(\mathrm{w} / \mathrm{m}^{\circ} \mathrm{C}\right)$ en $\mathrm{TN}$ & $T$ fusion $\left({ }^{\circ} \mathrm{C}\right)$ \\
\hline $\mathrm{TiN}$ & $18-21$ & 251 & 26,00 & - & 2950 \\
$\mathrm{TiC}$ & $28-35$ & 410 & 31,43 & 31,00 & 3067 \\
$\mathrm{WC}$ & 22 & 510 & - & 60,00 à $850{ }^{\circ} \mathrm{C}$ & 2870 \\
\hline
\end{tabular}

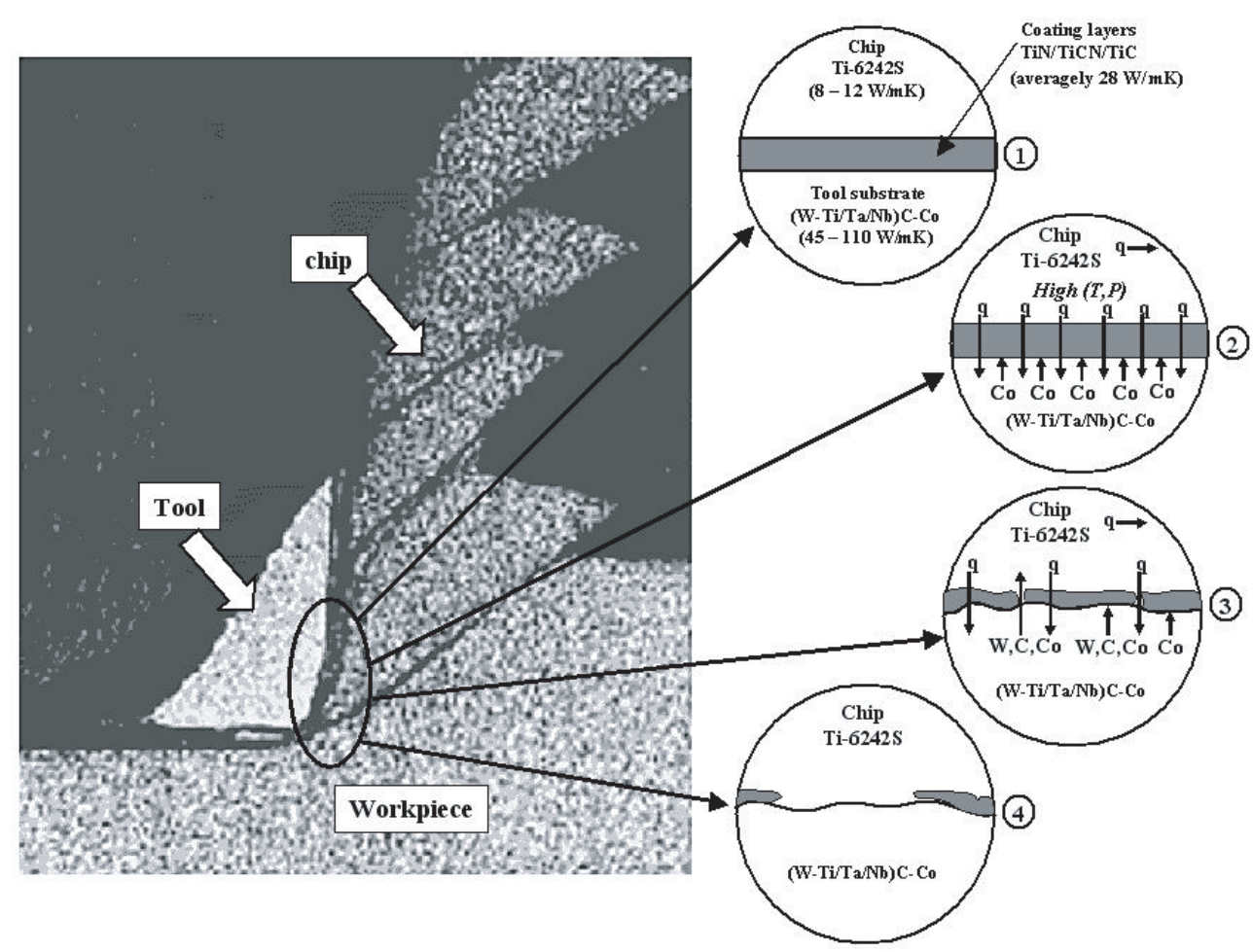

Fig. 7. Vue schématique du phénomène de délaminage des couches de revêtement.

plus important. Il reste toutefois à vérifier si la séquence d'empilement envisagée est réalisable techniquement, i.e. si les éléments chimiques constituant le revêtement sont compatibles chimiquement entre eux.

\subsection{Influence des paramètres thermiques sur le délaminage et leur évolution dans un contact à sec}

L'analyse précédente a montré une nette corrélation entre la température atteinte au niveau du contact et le processus de délaminage du revêtement. Cette analyse semble être consolidée par l'effet des paramètres thermiques sur la résistance à l'amorçage de fissures. La différence des paramètres thermiques de part et d'autre de l'interface n'est pas seulement due à la différence des propriétés thermomécaniques intrinsèques du matériau mais aussi à leur évolution avec le chargement imposé. L'examen des surfaces usées montre que l'ébrèchement excessif, l'écaillage et la rupture d'arête sont les principaux modes d'endommagement des outils carbures non revêtus (Figs. 1 et 2), tandis que pour les outils revêtus, la fissuration, la déformation plastique et le délaminage du revêtement prédominent (Figs. 3 et 4). Des études précédentes [14-21] sur l'usure d'un alliage de titane TA6V en contact glissant avec un acier ont montré que les zones affectées sont aussi des zones où la circulation de la chaleur se fait difficilement, (zone morte thermiquement). Aussi, la distribution de l'effusivité thermique a été montrée comme étant le paramètre fortement lié à l'usure dans le contact. L'effusivité thermique caractérise le degré de pénétration de la chaleur dans le matériau; elle est d'autant plus grande que la conductivité thermique est importante, mais une valeur élevée de l'effusivité thermique peut aussi être synonyme d'un matériau peu conducteur de chaleur avec une chaleur spécifique élevée (c'est-à-dire nécessitant beaucoup d'énergie pour que sa température augmente). Ce paramètre est donc un bon indicateur sur l'équilibre entre la capacité du matériau à conduire et à absorber la chaleur [19], elle est souvent donnée par l'expression suivante :

$$
E_{\mathrm{th}}=\sqrt{K \rho C}
$$

où $K$ est la conductivité thermique, $\rho$ la masse volumique, et $C$ la capacité calorifique massique du matériau. L'effusivité thermique peut aussi être interprétée comme 
l'aptitude du matériau à conduire et à diffuser la chaleur, elle peut donc être écrite sous la forme suivante :

$$
E_{\mathrm{th}}=\frac{K}{\sqrt{D}}
$$

où $D$ est le coefficient de diffusion thermique. Il est clair que dans le cas des équations (4) et (5), la conductivité thermique est le paramètre qui a le plus d'influence sur l'effusivité. En général, l'évolution de la conductivité en fonction de la température est représentée par des fonctions empiriques de types polynômes, exponentielles, bi-harmoniques, etc. Le choix d'une formulation mathématique particulière dépend essentiellement de la précision avec laquelle ce paramètre doit être identifié expérimentalement. Dans la suite de cette étude, une forme linéaire simple a été choisie :

$$
K(t)=K_{0}(1+\beta T)
$$

où $K_{0}$ est la conductivité thermique à la température ambiante et $\beta$ un coefficient caractéristique du matériau.

\subsection{Effet de la conductivité thermique sur le processus de délaminage du revêtement}

Pour mettre en évidence la relation entre usure (délaminage) et conductivité thermique, il est nécessaire de suivre l'évolution de ce dernier paramètre dans la Zone d'Outil Affectée (ZOA). Pour cela, plusieurs calculs par éléments-finis ont été conduits pour évaluer la distribution de la température dans cette zone. L'ensemble des nœuds de calcul a été sélectionné suivant quatre sous-couches délimitant la zone ZOA, voir figure 8a. Ces sous-couches ont été repérées sur le matériau suite aux observations microscopiques des facettes de l'outil présentant une usure importante. La figure $8 \mathrm{~b}$ illustre une vue schématique des nœuds de calcul superposés sur la surface réelle de l'outil. Deux sous-couches numérotées successivement 1 et 2 ont été choisies sur la face de dépouille, et deux autres numérotées 3 et 4 sur la face de coupe. La distance séparant respectivement les sous-couches 1-2 et 3-4 est de quelques micromètres. Sur les figures $9 \mathrm{a}$ et b, les températures locales correspondant aux différents nœuds de calcul sont représentées en fonction de la distance adimensionnelle $X / L$. $L$ est la distance entre le premier et le dernier nœud de chaque sous-couche. Les différents résultats de la figure 9 montrent que quelle que soit la sous-couche considérée, (sur la face de coupe ou la face de dépouille), la température présente un maximum situé à proximité de la pointe de l'outil (le point le plus chaud) ; elle diminue ensuite progressivement en s'y éloignant. En utilisant la référence [22] et les travaux de Salazar [23], la relation donnée par l'équation (6) a été appliquée pour déterminer l'évolution de la conductivité thermique effective du couple outil-revêtement en fonction de la température. La valeur du paramètre $\beta$ a été identifiée pour une gamme de températures allant de 20 à $1400{ }^{\circ} \mathrm{C}$.

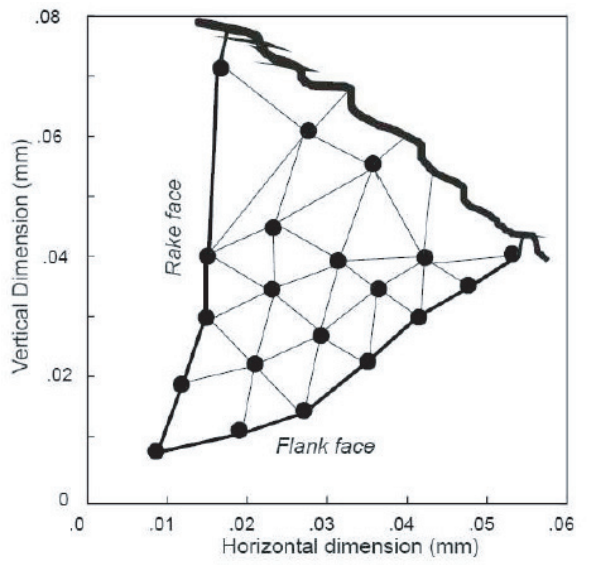

(a)
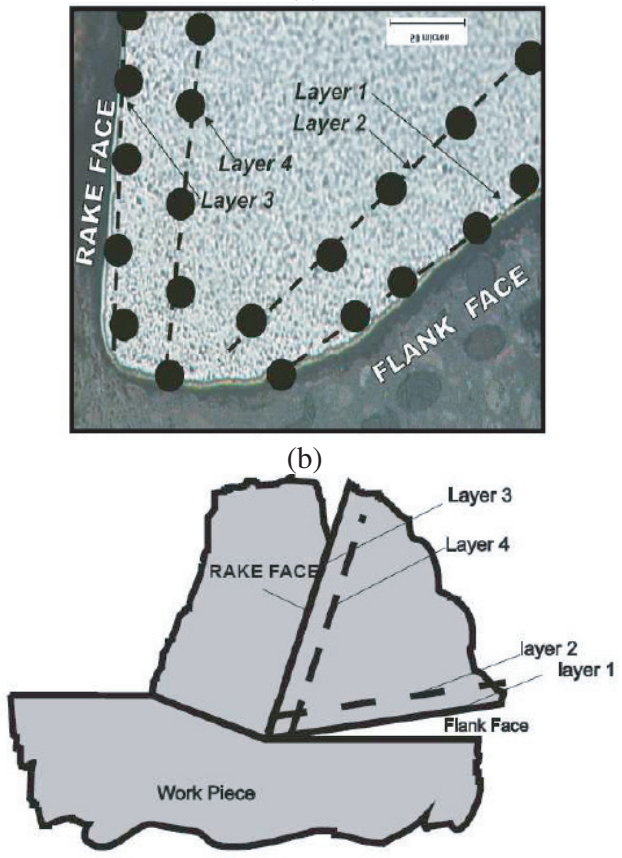

(c)

Fig. 8. Zone de de l'outil affectée par l'usure. (a) Maillage de la zone affectée par l'usure, (b) illustration schématique des nœuds de calcul sur l'outil réel et (c) position des souscouches 1, 2 sur la face de dépouille, 3 et 4 sur la face de coupe de l'outil.

Ainsi la conductivité effective identifiée est de la forme suivante :

$$
K(T)=45\left(1-507 \times 10^{-6} T\right)
$$

Les figures $10 \mathrm{a}$ et b montrent l'évolution du rapport des conductivités effectives $\frac{K(T)}{K_{0}}$, le long des quatre sous-couches sélectionnées précédemment. Les valeurs de ce rapport donnent la distribution locale de la conductivité du système (substrat+revêtement) dans la zone affectée par l'usure. Ces résultats montrent une conductivité relativement basse au voisinage de la pointe de l'outil, le matériau de l'outil évacue assez difficilement la chaleur à cet endroit. Comme il a été expliqué par ailleurs, la détérioration de la capacité du matériau à évacuer la 


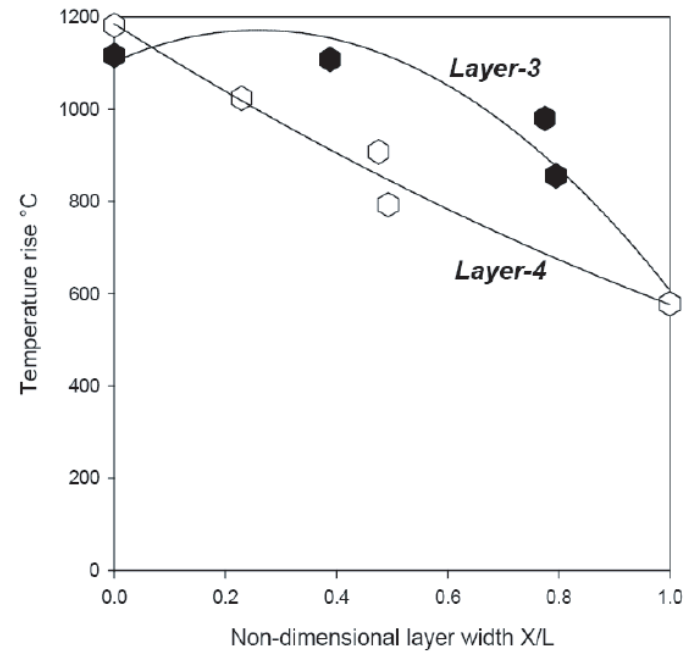

(a)

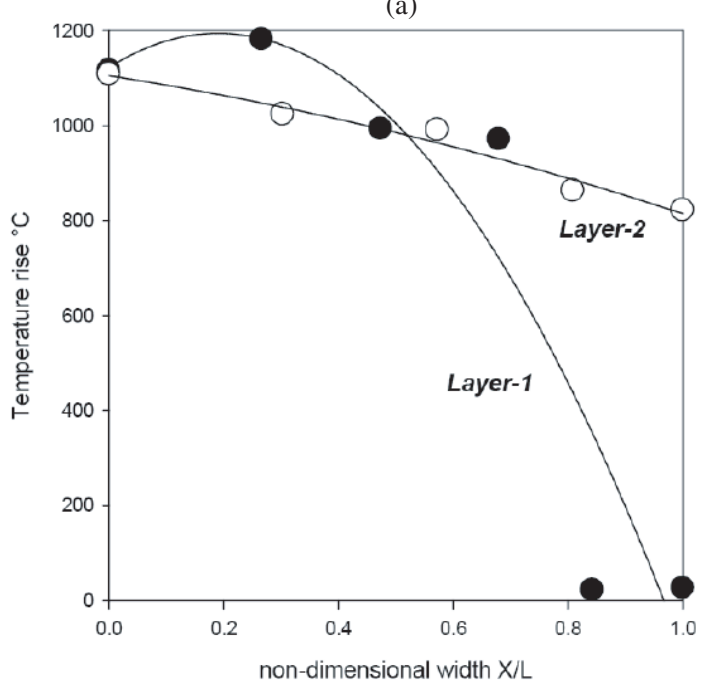

(b)

Fig. 9. Évolution de la température en fonction du paramètre adimensionnel $X / L, L$ est la distance entre le premier et le dernier nœud de chaque sous-couche $i(i=1-4)$, (a) souscouches 3 et 4 au niveau de la face de coupe, (b) sous-couches 1 et 2 au niveau de la face de dépouille (cf. Fig. 8).

charge thermique est la cause de l'activation de différents mécanismes d'usure dont le rôle est d'absorber l'excès d'énergie (réactions chimiques endothermiques, amorçage de fissures, diffusion, etc.).

Dans le cas de la figure 11, le rapport des conductivités $\frac{K(T)}{K_{0}}$ a été calculé en 8 endroits différents de la surface de l'outil où le délaminage a été observé. Il est intéressant de voir la différence de conductivités thermiques entre la surface et la subsurface de l'outil. L'arrangement en deux sous-couches des 8 nœuds de calcul est schématisé sur la surface réelle de l'outil neuf par la figure 11a et celle du même outil usé par la figure 11b. En analysant les résultats de la figure 11c, on remarque que les points I, II, III et IV se trouvant en surface se caractérisent par une plus faible conductivité que ceux en subsurface $\mathrm{I}^{\prime}$, II' $^{\prime}$ III' et IV', ( $\left.K_{\text {subsurface }}>K_{\text {surface }}\right)$. Cette différence de

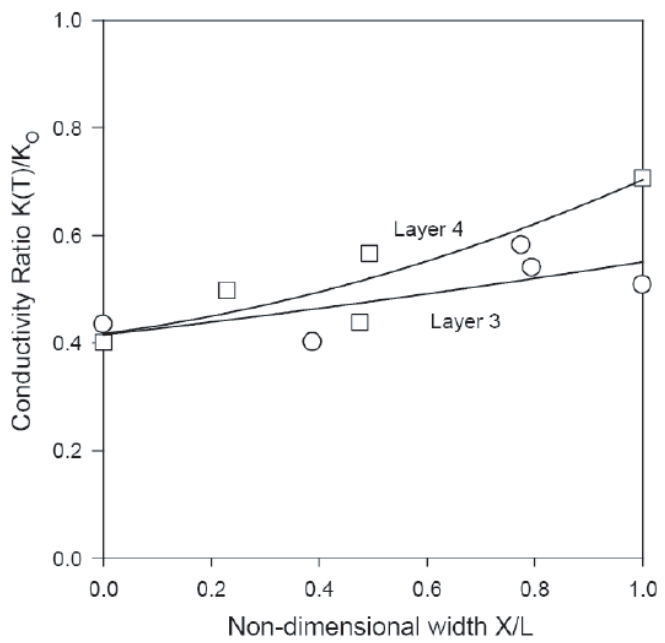

(a)

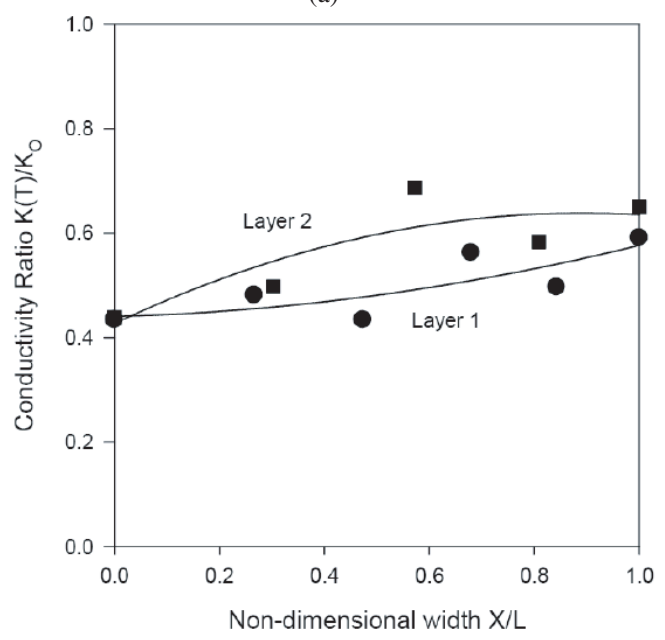

(a)

Fig. 10. Évolution de la conductivité thermique en fonction du paramètre adimensionnel $X / L, L$ est la distance entre le premier et le dernier nœud de chaque sous-couche $i(i=1-4)$, (a) sous-couches 3 et 4 au niveau de la face de coupe. (b) souscouches 1 et 2 au niveau de la face de dépouille (cf. Fig. 8).

conductivités effectives au niveau du contact outil-copeau va induire la dégradation de l'aptitude du matériau à évacuer la charge thermique, et à la diffuser (chute de l'effusivité thermique). Ceci va contribuer fortement à la création de zones dites « thermiquement mortes » à la surface de l'outil et provoquer fatalement un processus de délaminage de la première couche de revêtement.

\section{Conclusion}

La présente étude souligne l'importance de l'aspect thermique en usinage et son effet sur le comportement des revêtement d'outils face à l'usure. Les mécanismes d'endommagement identifiés sont principalement la fissuration et le délaminage des couches de revêtement. La chute de la conductivité thermique avec l'augmentation de la température limite localement l'aptitude du matériau à 


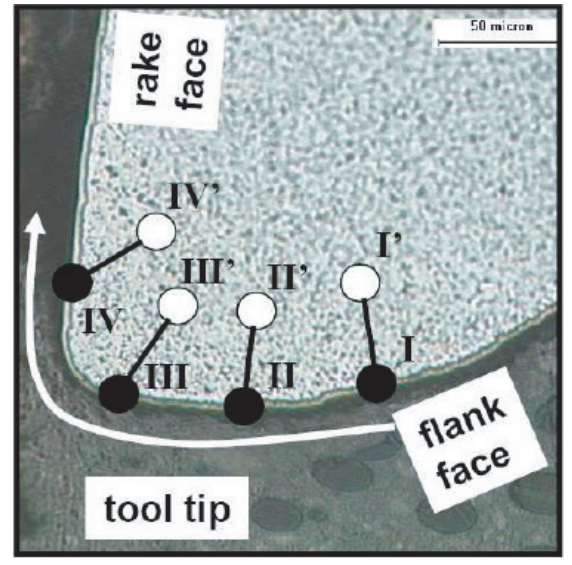

(b)

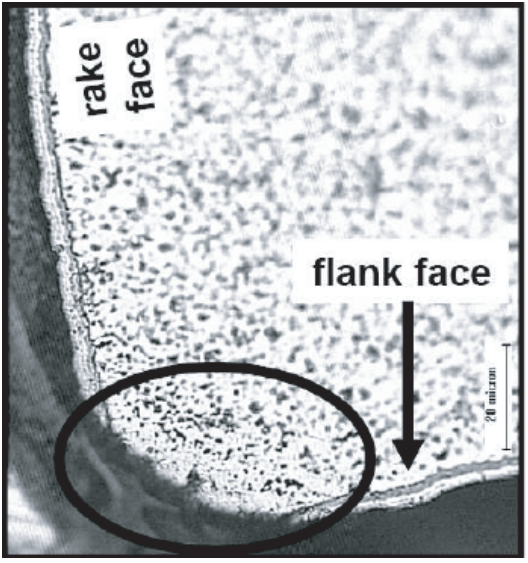

(b)

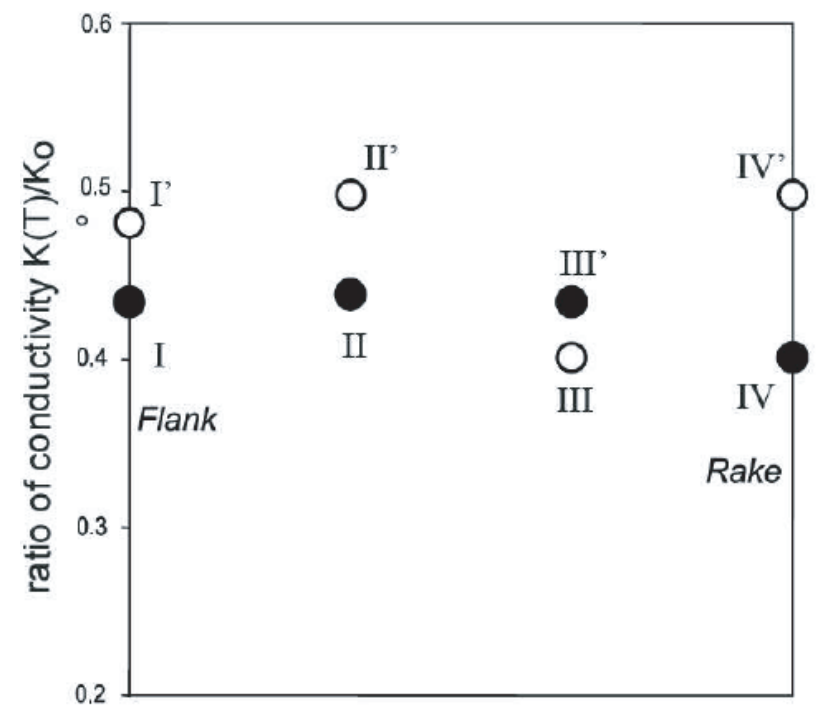

(c)

Fig. 11. Évolution du rapport des conductivités thermiques aux niveaux des 8 nœuds de calcul choisis dans la zone de l'outil affectée par l'usure.

dissiper la charge thermique. Plusieurs zones apparaissent alors aux niveaux de la face de coupe et de dépouille de l'outil où le transfert thermique se fait difficilement (zones mortes thermiquement). L'excès d'énergie thermique dans le matériau d'outil favorise ensuite l'activation de différents mécanismes dont le délaminage du revêtement. La maîtrise des paramètres thermiques et de leur évolution pendant une opération d'usinage peut alors aider à appréhender l'usure et à optimiser la résistance du revêtement au problème de délaminage.

Remerciements. Les auteurs remercient la Région Champagne-Ardenne et plus particulièrement Monsieur Jean-Paul Bachy (Président de la Région), d'avoir accorder le financement du séjour du Professeur H. A. Abdel Aâl d'une durée de six mois dans le cadre d'une collaboration entre l'ENSAM et l'Université du Wisconsin (USA), programme d'Accueil de Professeurs Étrangers, Contrat $\mathrm{N}^{\circ}$ DCPCR-ESR-0605-04.

\section{Références}

[1] C.H. Che Haron, A. Ginting, J.H. Goh, Wear of coated and uncoated carbides in turning tool steel, J. Materials Processing Technology 116 (2001) 49-54

[2] A. Jawaid, S. Sharif, S. Koksal, Evaluation of wear mechanisms of coated carbide tools when face milling titanium alloy, J. Materials Processing Technology 99 (2000) $266-274$

[3] M. Fitzsimmons, V.K. Sarin, Comparison of WCl-CH$\mathrm{H}$ and WF-CHH systems for growth of WC coatings, Surface and Coatings Technology 76-77 (1995) 250-255

[4] S.-S. Cho, K. Komvopoulos, Wear mechanisms of multilayer coated cemented carbide cutting tools, J. Tribology 119 (1997) 8-17

[5] W. Schintlmeister, W. Wallgram, J. Kanz, K. Gigl, Cutting tool materials coated by chemical vapour deposition, Wear 100 (1989) 153-169

[6] E.M. Trent, P.K. Wright, Metal Cutting (4th ed.), Butterworth-Heinemann, Boston, 2000 
[7] H.G. Prengel, P.C. Jindal, K.H. Wendt, A.T. Santhanam, P.L. Hegde, R.M. Penich, A new class of high performance PVD coatings for carbide cutting tools 139 (2001) 25-34

[8] A. Ginting, M. Nouari, Experimental and numerical studies on the performance of alloyed carbide tool in dry milling of aerospace materials, J. Machine Tools and Manufacture 46 (2006) 758-768

[9] M. Nouari, A. Ginting, Wear characteristics and performance of multi-layer CVD-coated alloyed carbide tool in dry end milling of titanium alloy, Surface and coating technology 200 (2006) 5663-5676

[10] A. Ginting, Tool-path generation for tapered machining features, M. Eng Thesis, Toyohashi University of Technology (TUT), Japan, 1999

[11] ISO 8688-2, Tool life testing in milling - Part 2, ed. Milling, 1989

[12] P.A. Dearnley, A.N. Grearson, Evaluation of principal wear mechanism of cemented carbides and ceramics used for machining titanium alloys IMI318, Material Sciences and Technology 2 (1986) 47-58

[13] M. Nouari, A. Molinari, Experimental verification of a diffusion tool wear model using a $42 \mathrm{CrMo} 4$ steel with an uncoated cemented tungsten carbide at various cutting speeds, Wear 259 (2005) 1151-1159

[14] H.A. Abdel-Aal, On the bulk temperatures of dry rubbing metallic solid pairs Int. Comm. Heat and Mass Transfer 26 (1999) 587-596
[15] H.A. Abdel-Aal, On the influence of tribo-induced superheating on protective layer formation in dry sliding of metallic pairs, Int. J. Th. Sci. 40 (2001) 571-580

[16] H.A. Abdel-Aal, On the connection of thermal dilatation to protective layer formation in the fretting of metallic tribo-specimens, Wear 247 (2001) 76-87

[17] H.A. Abdel-Aal, Correlating thermal dilatation to protective layer formation in fretting wear, Int. Commun. Heat and Mass Transfer 28 (2001) 97-106

[18] H.A. Abdel-Aal, On the influence of thermal properties on wear resistance of rubbing metals at elevated temperatures, J. Tribology 122 (2000) 657-660

[19] H.A. Abdel-Aal, The correlation between thermal property variation and high temperature wear transition of rubbing metals, Wear 237 (2000) 147-151

[20] H.A. Abdel-Aal, On the role of intrinsic material response in failure of tribo systems, Wear 259 (2005) 1372-1381

[21] H.A. Abdel-Aal, Error bounds of variable conductivity temperature estimates in frictionally heated contacts, Int. Comm. Heat and Mass Transfer 25 (1998) 99-108

[22] H.O. Pierson, Handbook of refractory carbides and nitrides, William Andrew Publishing, 2002

[23] A. Salazar, On thermal diffusivity, Eur. J. Phys. 24 (2003) 351-358

Retrouvez nos articles sur le site : www.edpsciences.org/meca 